\title{
A DIFFUSE INTERFACE MODEL FOR ALLOYS WITH MULTIPLE COMPONENTS AND PHASES*
}

\author{
HARALD GARCKE ${ }^{\dagger}$, BRITTA NESTLER $^{\ddagger}$, AND BJÖRN STINNER ${ }^{\dagger}$
}

\begin{abstract}
A nonisothermal phase field model for alloys with multiple phases and components is derived. The model allows for arbitrary phase diagrams. We relate the model to classical sharp interface models by formally matched asymptotic expansions. In addition we discuss several examples and relate our model to the ones already existing.
\end{abstract}

Key words. phase field models, sharp interface models, phase transitions, partial differential equations, alloy systems, matched asymptotic expansions

AMS subject classifications. 35K55, 82C26, 34E05, 35B25, 82C24

DOI. $10.1137 /$ S0036139902413143

1. Introduction. The phase field method is a powerful methodology to describe phase transition phenomena. The method has been used to describe solidification processes [7, 34] as well as microstructure evolution in solids [15] and liquid-liquid interfaces [28]. There are phase field models for pure substances [7, 34] and binary alloys [9, 21] for eutectic, peritectic, and monotectic systems [45, 31, 32, 33, 39]. Furthermore, the evolution of grain boundaries also can be modelled by phase field models or order parameter models $[12,16]$. For recent reviews of phase field methods we refer to $[13,5,14]$.

Traditionally the evolution of interfaces, such as the liquid-solid interface, has been modelled as a moving boundary problem. This means that pure phases are separated by a sharp interface. In the phases, partial differential equations, e.g., describing mass and heat diffusion, are solved. These equations are coupled by boundary conditions on the interface, such as the Stefan condition demanding energy balance and the Gibbs-Thomson equation. Across the sharp interface certain quantities (e.g., the heat flux, the concentration or the energy) may suffer jump discontinuities.

In phase field models the individual phases are distinguished by one or more socalled phase fields. In different phases the phase fields attain different values and interfaces are now modelled by a diffuse interface; i.e., the phase fields and all other quantities do not jump across an interface, but they change smoothly on a very thin transition layer (the diffuse interface). For example, for a solid-liquid phase transition we choose a phase field taking the value one in the solid and zero in the liquid; across an interface, the phase field varies smoothly from one to zero.

The use of diffuse interface models to describe interfacial phenomena dates back to van der Waals [42], Landau and Ginzburg [26], and Cahn and Hilliard [10]. In the theory of solidification this idea was introduced by Langer [27] and Caginalp [7]. Caginalp and Fife [8] used asymptotic expansions to relate the phase field models

*Received by the editors August 16, 2002; accepted for publication (in revised form) August 20, 2003; published electronically March 11, 2004. This work was supported by the DFG (Deutsche Forschungsgemeinschaft) through the Schwerpunktprogramm 1095 "Analysis, Modeling and Simulation of Multiscale Problems."

http://www.siam.org/journals/siap/64-3/41314.html

†NWF I - Mathematik, Universität Regensburg, 93040 Regensburg, Germany (harald.garcke@ mathematik.uni-regensburg.de, bjoern.stinner@mathematik.uni-regensburg.de).

${ }_{\ddagger}^{\ddagger}$ Fachbereich Informatik, FH Karlsruhe, Moltkestraße 30, 76133 Karlsruhe, Germany (britta. nestler@fh-karlsruhe.de). 
proposed by Langer to classical free boundary problems in the sharp interface limit. This relation has also been rigorously established for some cases (see, for example, $[38,41]$ and the references therein).

Since the original phase field model is not derived from thermodynamical principles, a number of so-called thermodynamically consistent phase field models were proposed in the 1990s (see Penrose and Fife [34], Alt and Pawlow [2], Wang et al. [44]). All of these models guarantee a positive entropy production.

The classical asymptotics leads to restrictions on parameters which often makes it difficult to perform practical computations of realistic solidification processes. This is particularly true in the regime of small undercooling. In recent years Karma and Rappel $[23,24]$ (see also $[25,1,30]$ ) used the so-called thin interface asymptotics to realize numerical simulations in this regime. There, the Gibbs-Thomson equation is approximated to a higher order and the temperature profile in the interfacial region is recovered with a higher accuracy when compared to the classical asymptotics. Further numerical simulations (see $[35,36,37]$ ) confirm the superiority of this approach in the case of small undercooling.

So far, generalizing this approach to more general situations (see the discussion in [25]) and, in particular, extending the approach to phase field systems handling multiple phases are still an open problem. Therefore, as a first step, we apply classical sharp interface asymptotics to handle general systems with multiple phases and components. The task of making this approach more efficient by the use of thin interface asymptotics is left to further research.

The aim of this paper is to derive a phase field model that

- is thermodynamically consistent,

- allows for an arbitrary number of phases and components,

- is defined solely via the bulk free energies of the individual phases, the surface energy densities (surface entropy densities, respectively) of the interfaces, and diffusion and mobility coefficients, and

- yields classical moving boundary problems in the sharp interface limit.

The third requirement enables us to define the full set of phase field evolution equations by quantities which (in principal) can be measured. Since the bulk free energies determine the phase diagrams (see, e.g., Chalmers [11], Haasen [22]) our model can be used to model phase transitions for arbitrary phase diagrams. We note that in a multi-phase field model computing the surface free energy densities (or surface entropy densities) is difficult. Here one can make use of the studies by Garcke, Nestler, and Stoth [18], in which free energies for phase field methods with good calibration properties have been developed. This means that for given surface free energies (also called surface tensions) one can calibrate the parameters in the free energies of the phase field model in such a way that the sharp interface limit is defined via the given surface tensions. In particular the sharp interface problem is defined with the help of the surface free energies.

In the following section we introduce the phase field model in its full generality and state the corresponding sharp interface model. In section 3 we give examples and relate the model we propose to models already existing in the literature. Furthermore, we discuss a variety of different applications for the new model. Due to its general formulation, the model has the capability to describe phase transformation processes in nonisothermal multicomponent alloys as well as in grain structure evolution. Different phases and different crystal orientations can be distinguished at the same time by an appropriate choice of the phase field variables. This allows us to treat 
effects occurring on different length scales such as eutectic grains and interdendritic structures.

Finally, we show in section 4 via formally matched asymptotic expansions that the phase field model yields the sharp interface model in the limit when the interfacial thickness tends to zero.

2. The models. We consider a domain $\Omega \subset \mathbf{R}^{d}, d \in\{1,2,3\}$, and we assume that the system has $N$ components with $M$ different phases possible.

2.1. The phase field model. The phase field model is based on an entropy functional of the form

$$
S(e, c, \phi)=\int_{\Omega}\left(s(e, c, \phi)-\left(\varepsilon a(\phi, \nabla \phi)+\frac{1}{\varepsilon} w(\phi)\right)\right) d x .
$$

We assume that the bulk entropy density $s$ depends on the internal energy density $e$, the concentrations of the $N$ components $c_{i}, 1 \leq i \leq N$, and the phase field variable $\phi=\left(\phi_{\alpha}\right)_{\alpha=1}^{M}$. The variable $\phi_{\alpha}$ denotes the local fraction of phase $\alpha$, and we require that the concentrations of the components and the phase field variables fulfill the constraints

$$
\sum_{i=1}^{N} c_{i}=1, \quad \sum_{\alpha=1}^{M} \phi_{\alpha}=1 .
$$

It will be convenient to use the free energy as a thermodynamical potential. We therefore postulate the Gibbs relation

$$
d f=-s d T+\sum_{i} \mu_{i} d c_{i}+\sum_{\alpha} r_{\alpha} d \phi_{\alpha}
$$

(see Alt and Pawlow [3], who show that the Gibbs relation is a consequence of the entropy principle). Here, $T$ is the temperature, $\mu_{i}=f_{,_{i}}$ are the chemical potentials, and $r_{\alpha}=f_{, \phi_{\alpha}}$ are potentials due to the appearance of different phases.

We set

$$
e=f+s T \text {, }
$$

and hence

$$
\begin{aligned}
& d e=T d s+\sum_{i} \mu_{i} d c_{i}+\sum_{\alpha} r_{\alpha} d \phi_{\alpha}, \\
& d s=\frac{1}{T} d e-\sum_{i} \frac{\mu_{i}}{T} d c_{i}-\sum_{\alpha} \frac{r_{\alpha}}{T} d \phi_{\alpha} .
\end{aligned}
$$

If we interpret $s$ as a function of $(e, c, \phi)$, then we have

$$
s_{, e}=\frac{1}{T}, \quad s_{, c_{i}}=\frac{-\mu_{i}}{T}, \quad s_{, \phi_{\alpha}}=\frac{-r_{\alpha}}{T} .
$$

Later it will be convenient to switch among the variables $(T, c, \phi),(e, c, \phi),(T, \mu, \phi)$, and $\left(-\frac{1}{T}, \frac{1}{T} \mu, \phi\right)$, and we therefore assume for the rest of this paper that

- $c \mapsto f(T, c, \phi)$ is strictly convex,

- $T \mapsto f(T, c, \phi)$ is strictly concave. 
This will make the above exchanges of variables possible.

We note that given the free energy densities of the pure phases, we obtain the total free energy as a suitable interpolation of the free energies $f_{\alpha}$, i.e., such that $f\left(T, c, e_{\alpha}\right)=f_{\alpha}(T, c)$, with $e_{\alpha}$ being the $\alpha$ th coordinate vector.

So far we have neglected interfacial effects. The thermodynamics of the interface gives additional contributions to entropy and free energy. Let us first consider how interfacial contributions are accounted for in a sharp interface model. Let $\Gamma_{\alpha \beta}$ denote an interface between phases $\alpha$ and $\beta$ and let $\nu_{\alpha \beta}$ denote the unit normal at $\Gamma_{\alpha \beta}$ pointing into the $\beta$-phase. Then in sharp interface models an interfacial term

$$
-\sum_{\substack{\alpha<\beta \\ \alpha, \beta=1}}^{M} \int_{\Gamma_{\alpha \beta}} \gamma_{\alpha \beta}\left(\nu_{\alpha \beta}\right) d \mathcal{H}^{d-1}
$$

with a positive function $\gamma_{\alpha \beta}$ on $S^{d-1}$ is added to the entropy (see [29], [43]). The notation $d \mathcal{H}^{d-1}$ indicates integration with respect to the $(d-1)$-dimensional surface measure.

In diffuse interface models the surface entropy functional (6) is replaced by a Ginzburg-Landau type functional of the form

$$
-\int_{\Omega}\left(\varepsilon a(\phi, \nabla \phi)+\frac{1}{\varepsilon} w(\phi)\right) d x .
$$

Here, $a$ is the gradient energy density which is assumed to be homogeneous of degree two in the second variable; i.e.,

$$
a(\phi, \eta X)=\eta^{2} a(\phi, X) \quad \forall(\phi, X) \in \mathbf{R}^{M} \times \mathbf{R}^{d \times M} \text { and } \forall \eta \in \mathbf{R}^{+},
$$

and $w$ is a nonconvex function with exactly $M$ global minima at the points $e_{\beta}=$ $\left(\delta_{\alpha, \beta}\right)_{\alpha=1}^{M}, 1 \leq \beta \leq M$, with $w\left(e_{\alpha}\right)=0$. It has been shown under appropriate assumptions on $a$ that the functional (7) converges to the perimeter functional (6) when $\varepsilon$ converges to zero. We refer to [18], [19] and section 3 for appropriate choice of $a$ and $w$. We assume in this paper that $a$ and $w$ and, hence, the interfacial contributions to the entropy, do not depend on $(T, c)$, but these dependences can be included, leading to a much more complicated model.

Our goal is to derive balance equations,

$$
\begin{aligned}
\partial_{t} e & =-\nabla \cdot J_{0} \quad & & (\text { energy balance), } \\
\partial_{t} c_{i} & =-\nabla \cdot J_{i} & & \text { (mass balances, } i=1, \ldots, N),
\end{aligned}
$$

that are coupled to

$$
\partial_{t} \phi_{\alpha}=\text { right-hand side }(R H S)
$$

in such a way that the second law of thermodynamics is fulfilled in an appropriate local version. Here, $J_{0}$ is the energy flux and $J_{1}, \ldots, J_{N}$ are the fluxes of the components $c_{1}, \ldots, c_{N}$. In order to derive appropriate expressions for the fluxes $J_{0}, \ldots, J_{N}$, we use the generalized thermodynamic potentials (compare $(5 \mathrm{~b})) \frac{\delta S}{\delta e}=\frac{1}{T}$ and $\frac{\delta S}{\delta c_{i}}=\left(\frac{-\mu_{i}}{T}\right)$, which will drive the evolution. Now we appeal to nonequilibrium thermodynamics and postulate that the fluxes are linear functions of the thermodynamic driving forces 
$\nabla \frac{\delta S}{\delta e}, \nabla \frac{\delta S}{\delta c_{1}}, \ldots, \nabla \frac{\delta S}{\delta c_{N}}$ to obtain

$$
\begin{aligned}
J_{0} & =L_{00}(T, c, \phi) \nabla \frac{\delta S}{\delta e}+\sum_{j=1}^{N} L_{0 j}(T, c, \phi) \nabla \frac{\delta S}{\delta c_{j}} \\
& =L_{00}(T, c, \phi) \nabla \frac{1}{T}+\sum_{j=1}^{N} L_{0 j}(T, c, \phi) \nabla \frac{-\mu_{j}}{T}, \\
J_{i} & =L_{i 0}(T, c, \phi) \nabla \frac{\delta S}{\delta e}+\sum_{j=1}^{N} L_{i j}(T, c, \phi) \nabla \frac{\delta S}{\delta c_{j}} \\
& =L_{i 0}(T, c, \phi) \nabla \frac{1}{T}+\sum_{j=1}^{N} L_{i j}(T, c, \phi) \nabla \frac{-\mu_{j}}{T}
\end{aligned}
$$

with mobility coefficients

$$
\left(L_{i j}\right)_{i, j=0, \ldots, N} .
$$

To fulfill the constraint $\sum_{i=1}^{N} c_{i}=1$ during the evolution, we assume

$$
\sum_{i=1}^{N} L_{i j}=0, \quad j=0, \ldots, N,
$$

which implies $\sum_{i=1}^{N} J_{i}=0$, and, hence, $\partial_{t}\left(\sum_{i=1}^{N} c_{i}\right)=\nabla \cdot\left(\sum_{i=1}^{N} J_{i}\right)=0$. We further assume that $L$ is symmetric (Onsager relations). In addition, $L$ is assumed to be positive semidefinite; i.e.,

$$
\sum_{i, j=0}^{N} L_{i j} \xi_{i} \xi_{j} \geq 0 \quad \forall \xi=\left(\xi_{0}, \ldots, \xi_{N}\right) \in \mathbf{R}^{N+1} .
$$

This condition will later ensure that an entropy inequality is satisfied. We note that we include cross effects between mass and energy diffusion in the model. One can neglect them by setting $L_{i 0}=0$ and $L_{0 j}=0$ for all $i, j \in\{1, \ldots, N\}$.

For the nonconserved phase field variables $\phi_{1}, \ldots, \phi_{M}$, we assume that the evolution is such that the system locally tends to maximize entropy conserving concentration and energy at the same time. Therefore we postulate

$$
\begin{aligned}
\omega \varepsilon \partial_{t} \phi_{\alpha} & =\frac{\delta S}{\delta \phi_{\alpha}}-\lambda \\
& =\varepsilon\left(\nabla \cdot a_{, X_{\alpha}}(\phi, \nabla \phi)-a_{, \phi_{\alpha}}(\phi, \nabla \phi)\right)-\frac{1}{\varepsilon} w_{, \phi_{\alpha}}(\phi)-\frac{f_{, \phi_{\alpha}}}{T}-\lambda,
\end{aligned}
$$

where we denote with $a_{, X_{\alpha}}$ the derivative with respect to the variables corresponding to $\nabla \phi_{\alpha} . \omega$ is (in this paper) a constant kinetic coefficient and $\lambda$ is an appropriate Lagrange multiplier such that the constraint $\sum_{\alpha=1}^{M} \phi_{\alpha}=1$ is satisfied; i.e.,

$$
\lambda=\frac{1}{M} \sum_{\alpha}\left[\varepsilon\left(\nabla \cdot a_{, X_{\alpha}}-a_{, \phi_{\alpha}}\right)-\frac{1}{\varepsilon} w_{, \phi_{\alpha}}-\frac{f_{, \phi_{\alpha}}}{T}\right] .
$$

Relevant for the dynamics are the variational derivatives of $S$ that take the constraints (2) into account. We can therefore reformulate (9b) and (12) in terms of 
the projection of $\left(\frac{\delta S}{\delta e}, \frac{\delta S}{\delta c_{j}}, \frac{\delta S}{\delta \phi_{\alpha}}\right)$ onto the tangent space of the linear subspace whose elements satisfy the constraints. Defining

$$
\Sigma^{K}=\left\{d \in \mathbf{R}^{K}: \quad \sum_{k=1}^{K} d_{k}=1\right\}
$$

and its tangent space

$$
T \Sigma^{K}=\left\{d \in \mathbf{R}^{K}: \quad \sum_{k=1}^{K} d_{k}=0\right\}
$$

the constraints (2) read as $c \in \Sigma^{N}$ and $\phi \in \Sigma^{M}$. In the following, $P^{K}$ will denote the projection onto $T \Sigma^{K}$. Then the relevant quantities for the definition of the fluxes are

$$
\left(P^{N}\left(-\frac{1}{T} \mu\right)\right)_{i}=-\frac{1}{T}\left(\mu_{i}-\frac{1}{N} \sum_{j} \mu_{j}\right)=-\frac{1}{T} \frac{1}{N} \sum_{j}\left(\mu_{i}-\mu_{j}\right),
$$

whereas there are no changes to $\frac{\delta S}{\delta e}$. We note that the quantities

$$
\bar{\mu}_{i}=\frac{1}{N} \sum_{j}\left(\mu_{i}-\mu_{j}\right)
$$

can be interpreted as generalized chemical potential differences. For two components we obtain $\bar{\mu}_{1}=\left(\mu_{1}-\mu_{2}\right) / 2$, i.e., the usual chemical potential difference multiplied by the factor $1 / 2$.

With the above notation we can rewrite the fluxes as

$$
\begin{gathered}
J_{0}=L_{00}(T, c, \phi) \nabla \frac{1}{T}+\sum_{j=1}^{N} L_{0 j}(T, c, \phi) \nabla \frac{-\bar{\mu}_{j}}{T}, \\
J_{i}=L_{i 0}(T, c, \phi) \nabla \frac{1}{T}+\sum_{j=1}^{N} L_{i j}(T, c, \phi) \nabla \frac{-\bar{\mu}_{j}}{T} .
\end{gathered}
$$

Similarly we can rewrite (12) as

$$
\omega \varepsilon \partial_{t} \phi=P^{M}\left[\varepsilon\left(\nabla \cdot a_{, X}(\phi, \nabla \phi)-a_{, \phi}(\phi, \nabla \phi)\right)-\frac{1}{\varepsilon} w_{, \phi}(\phi)-\frac{f_{, \phi}}{T}\right] .
$$

Altogether the total entropy density is given by

$$
\text { bulk entropy }+ \text { surface entropy }=s(e, c, \phi)-\left(\varepsilon a(\phi, \nabla \phi)+\frac{1}{\varepsilon} w(\phi)\right),
$$

and a straightforward computation shows (setting $\mu_{0}=-1$ )

$$
\begin{aligned}
\partial_{t}(\text { entropy })= & \partial_{t}\left(s(e, c, \phi)-\varepsilon a(\phi, \nabla \phi)-\frac{1}{\varepsilon} w(\phi)\right) \\
= & \sum_{i, j=0}^{N} \nabla \frac{-\mu_{i}}{T} \cdot L_{i j} \nabla \frac{-\mu_{j}}{T}-\nabla \cdot\left(\sum_{i, j=0}^{N} \frac{-\mu_{i}}{T} L_{i j} \nabla \frac{-\mu_{j}}{T}\right) \\
& +\omega \varepsilon \sum_{\alpha}\left(\partial_{t} \phi_{\alpha}\right)^{2}-\varepsilon \sum_{\alpha} \nabla \cdot\left(a_{, X_{\alpha}} \partial_{t} \phi_{\alpha}\right) \\
\geq & -\nabla \cdot\left(\sum_{i=0}^{N} \frac{-\mu_{i}}{T} J_{i}-\varepsilon \sum_{\alpha=1}^{M} a_{, X_{\alpha}} \partial_{t} \phi_{\alpha}\right) .
\end{aligned}
$$


The above inequality shows that the local entropy production is positive where the entropy flux $J_{s}$ is given by

$$
J_{s}=\sum_{i=0}^{N}\left(\frac{-\mu_{i}}{T} J_{i}\right)-\varepsilon \sum_{\alpha=1}^{M} a_{, p_{\alpha}} \partial_{t} \phi_{\alpha} .
$$

The first term represents the entropy flux due to mass and energy diffusion, and the second one is due to moving phase boundaries (compare [2]). We refer to Alt and Pawlow [3], who show that for conserved phase fields (they call them order parameters) either the energy flux or the entropy flux has to depend on $\partial_{t} \phi$ in order to describe phase transitions.

2.2. The sharp interface model. In section 4 we use the method of asymptotic expansions to relate the phase field model of the previous subsection to the sharp interface model which we state in the following. We obtain that when the domain $\Omega$ is separated in phase regions $\Omega_{1}, \ldots, \Omega_{M}$ occupied by the pure phases $1, \ldots, M$ such that in every phase $\Omega_{\alpha}, \alpha=1, \ldots, M$, the following evolution equations hold:

$$
\begin{aligned}
& \partial_{t} e^{\alpha}=-\nabla \cdot\left(L_{00}^{\alpha}\left(T^{\alpha}, c^{\alpha}\right) \nabla \frac{1}{T^{\alpha}}-\sum_{j=1}^{N} L_{0 j}^{\alpha}\left(T^{\alpha}, c^{\alpha}\right) \nabla \frac{\mu_{j}^{\alpha}}{T^{\alpha}}\right) \quad(\text { energy balance), } \\
& \partial_{t} c_{i}^{\alpha}=-\nabla \cdot\left(L_{i 0}^{\alpha}\left(T^{\alpha}, c^{\alpha}\right) \nabla \frac{1}{T^{\alpha}}-\sum_{j=1}^{N} L_{i j}^{\alpha}\left(T^{\alpha}, c^{\alpha}\right) \nabla \frac{\mu_{j}^{\alpha}}{T^{\alpha}}\right) \forall i \text { (mass balances). }
\end{aligned}
$$

These equations can be formulated in the variables $(T, \mu)$ (in which case the internal energy $e^{\alpha}$ and the concentrations $c^{\alpha}$ are given as $e^{\alpha}=e^{\alpha}\left(T^{\alpha}, \mu^{\alpha}\right)$ and $c^{\alpha}=$ $c^{\alpha}\left(T^{\alpha}, \mu^{\alpha}\right)$ ) or, more commonly, in the variables $(T, c)$ (in which case the internal energy $e^{\alpha}$ and the chemical potentials $\mu^{\alpha}$ are given as $e^{\alpha}=e^{\alpha}\left(T^{\alpha}, c^{\alpha}\right)$ and $\left.\mu^{\alpha}=c^{\alpha}\left(T^{\alpha}, c^{\alpha}\right)\right)$.

On a (smooth) boundary $\Gamma_{\alpha \beta}$ between two phases $\alpha$ and $\beta$ we have (assuming an isotropic surface energy)

$$
\begin{aligned}
& T^{\alpha}=T^{\beta}=: T \quad \text { (continuity of temperature), } \\
& \bar{\mu}_{i}^{\alpha}=\bar{\mu}_{i}^{\beta}=: \bar{\mu}_{i} \quad \forall i \quad \text { (continuity of chemical potentials), } \\
& {[e]_{\alpha}^{\beta} v=\left[J_{0}\right]_{\alpha}^{\beta} \cdot \nu \quad \text { (energy balance), }} \\
& {\left[c_{i}\right]_{\alpha}^{\beta} v=\left[J_{i}\right]_{\alpha}^{\beta} \cdot \nu \quad \forall i \quad \text { (mass balances), }} \\
& m_{\alpha \beta} v=\gamma_{\alpha \beta} \kappa+\frac{[f]_{\alpha}^{\beta}-\sum_{i} \bar{\mu}_{i}\left[c_{i}\right]_{\alpha}^{\beta}}{T} \text { (Gibbs-Thomson relation). }
\end{aligned}
$$

Here, $\nu=\nu_{\alpha \beta}$ is the unit normal pointing into $\beta, v$ is the speed of $\Gamma$ in this direction, and $\kappa$ is the mean curvature. The quantities

$$
\bar{\mu}_{i}^{\alpha}=\mu_{i}^{\alpha}-\frac{1}{N} \sum_{j=1}^{N} \mu_{j}^{\alpha}=\frac{1}{N} \sum_{j=1}^{N}\left(\mu_{i}^{\alpha}-\mu_{j}^{\alpha}\right),
$$

where $\mu_{i}^{\alpha}=f_{, c_{i}}^{\alpha}(T, c)$ are the generalized chemical potential differences in phase $\alpha$, and $[\cdot]_{\alpha}^{\beta}$ denotes the jump of the quantity in the brackets across the interface. The quantity 
$\gamma_{\alpha \beta}$ is the surface entropy density and the relation between the surface entropy and the entropy density in the phase field model is given by

$$
\gamma_{\alpha \beta}=\inf _{p}\left\{2 \int_{-1}^{1} \sqrt{w(p)} \sqrt{a\left(p, p^{\prime} \otimes \nu\right)}\right\},
$$

where the infimum is taken over all Lipschitz continuous functions $p$ connecting the minima of $w$ corresponding to the phases adjacent to the interface, i.e., $p(-1)=e_{\alpha}$ and $p(1)=e_{\beta}$. The kinetic coefficient $m_{\alpha \beta}$ can also be expressed in terms of the minimizer $p$ (see [17]).

In general, $a$ and $w$ might depend on temperature and on the concentrations leading to a temperature- and concentration-dependent surface entropy in the sharp interface limit. In this case, the surface terms would also enter the internal energy.

For a thin interface analysis of a partially linearized model for pure substances we refer to [30]. Performing a thin interface analysis for our model would require studying higher order corrections of fields like $s, f, T$, and $c$ in the interface region. We do not pursue this issue further at this stage.

We note that the Gibbs-Thomson equation can be derived by locally maximizing entropy, conserving concentration and energy at the same time. For a stationary flat interface the equations (17), (18), and (21) yield the classical equilibrium for phase boundaries. The equilibrium condition at a flat boundary at rest separating phases $\alpha$ and $\beta$ is

$$
\bar{\mu}_{i}^{\alpha}=\bar{\mu}_{i}^{\beta} \quad \text { for all } \quad i=1, \ldots, N .
$$

In addition the temperature has to be the same and (see (21))

$$
[f]_{\alpha}^{\beta}-\sum_{i} \bar{\mu}_{i}\left[c_{i}\right]_{\alpha}^{\beta}=0 .
$$

For $M$ phases to be in equilibrium we therefore have $(N+1)(M-1)$ conditions. For each phase we can choose $N-1$ components and the temperature. All together there are

$$
M N-(N+1)(M-1)=N-M+1
$$

degrees of freedom. This is the Gibbs phase rule. We note that for two component systems the equilibrium conditions between two phases lead to the well-known common tangent construction.

Finally, at triple junctions where three phases $\alpha, \beta$, and $\delta$ meet, a force balance of the form

$$
\gamma_{\alpha \beta} \tau_{\alpha \beta}+\gamma_{\beta \delta} \tau_{\beta \delta}+\gamma_{\delta \alpha} \tau_{\delta \alpha}=0
$$

has to hold (compare [19]). Here, $\tau_{\alpha \beta}, \tau_{\beta \delta}$, and $\tau_{\delta \alpha}$ are the tangents to the interfaces $\Gamma_{\alpha \beta}, \Gamma_{\beta \delta}$, and $\Gamma_{\delta \alpha}$. All are assumed to either point in the direction of the triple junction or point away from the triple junction at the same time. It can be easily seen that this force balance is equivalent to certain angle conditions at the triple junction.

In the appendix we will demonstrate that the entropy does not decrease for solutions of the above problem. In particular, for a closed system we obtain, using 
appropriate transport theorems and assuming $m \geq 0$ and $L=\left(L_{i j}\right)_{i, j=1, \ldots, N}$ is positive semidefinite, the following:

$$
\begin{aligned}
\frac{d}{d t}\left(\int_{\Omega} s(e, c) d x-\int_{\Gamma} \gamma d \mathcal{H}^{d-1}\right)= & \int_{\Omega}\left(\nabla \frac{1}{T} \cdot J_{0}+\sum_{i} \nabla \frac{-\bar{\mu}_{i}}{T} \cdot J_{i}\right) d x \\
& +\int_{\Gamma} m v^{2} d \mathcal{H}^{d-1} \quad \geq 0
\end{aligned}
$$

where the integral over $\Gamma$ is an integral over all possible interfaces.

3. Examples. In this section we will first demonstrate that the phase field method is able to model systems with a very general class of phase diagrams. In the way it is formulated, the model can describe systems with concave entropies $s_{\alpha}(e, c)$ in the pure phases. This corresponds to free energies $f_{\alpha}(T, c)$ which are convex in $c$ and concave in $T$. In the case where $f(T, c)$ is not convex in the variable $c$, the free energy needs to contain gradients of the concentrations (as in the Cahn-Hilliard model).

We will first give a rather general example, which already covers most examples in practice, and then discuss relations to existing models and possible partial linearizations of the system.

3.1. Possible choices of the free energy. Choosing the phase field $\phi$ such that $\phi=e_{M}$ corresponds to the liquid phase, we define bulk free energies for the individual phases by

$$
f_{\alpha}(T, c)=\sum_{i=1}^{N}\left(c_{i} L_{i}^{\alpha} \frac{T-T_{i}^{\alpha}}{T_{i}^{\alpha}}+\frac{R}{v_{m}} T c_{i} \ln \left(c_{i}\right)\right)-c_{v} T(\ln (T)-1)
$$

with $L_{i}^{M}=0$, and $L_{i}^{\alpha}, i=1, \ldots, N, \alpha=1, \ldots, M-1$, being the latent heat per unit volume of the phase transition from phase $\alpha$ to the liquid phase of the pure component $i$. Furthermore, $T_{i}^{\alpha}, i=1, \ldots, N, \alpha=1, \ldots, M-1$, is the melting temperature of the $i$ th component in the phase $\alpha$, and $c_{v}$ is the specific heat, which is assumed to be independent of $c$ and $\phi$; the molar volume $v_{m}$ is supposed to be a constant, and $R$ is the gas constant. Then we define the total free energy density as follows:

$$
\begin{aligned}
f(T, c, \phi):= & \sum_{\alpha=1}^{M} \sum_{i=1}^{N}\left(c_{i} L_{i}^{\alpha} \frac{T-T_{i}^{\alpha}}{T_{i}^{\alpha}} h\left(\phi_{\alpha}\right)\right) \\
& +\sum_{i=1}^{N}\left(\frac{R}{v_{m}} T c_{i} \ln \left(c_{i}\right)\right)-c_{v} T(\ln (T)-1),
\end{aligned}
$$

where $h$ is a monotone function on $[0,1]$ that satisfies $h(0)=0$ and $h(1)=1$. Examples are $h(\phi)=\phi$ and $h(\phi)=\phi^{2}(3-2 \phi)$. The last one has the property $h^{\prime}(0)=h^{\prime}(1)=0$ which is suitable for phase field models as we will see below. With this choice of $h$ the function $f$ is an interpolation of the individual free energy densities $f_{\alpha}$.

We can calculate

$$
s=-f_{, T}=-\sum_{\alpha=1}^{M} \sum_{i=1}^{N}\left(c_{i} \frac{L_{i}^{\alpha}}{T_{i}^{\alpha}} h\left(\phi_{\alpha}\right)\right)-\sum_{i=1}^{N}\left(\frac{R}{v_{m}} c_{i} \ln \left(c_{i}\right)\right)+c_{v} \ln (T),
$$


so that

$$
e=f+T s=-\sum_{\alpha=1}^{M} \sum_{i=1}^{N}\left(c_{i} L_{i}^{\alpha} h\left(\phi_{\alpha}\right)\right)+c_{v} T .
$$

We note that if $L_{i}^{\alpha}=L^{\alpha}$ for all components $i$, then $e$ does not depend on $c$. The chemical potentials are given as

$$
\mu_{i}(T, c, \phi)=f_{, c_{i}}(T, c, \phi)=\sum_{\alpha=1}^{M}\left(L_{i}^{\alpha} \frac{T-T_{i}^{\alpha}}{T_{i}^{\alpha}} h\left(\phi_{\alpha}\right)\right)+\frac{R}{v_{m}} T\left(\ln \left(c_{i}\right)+1\right) .
$$

Expressions for the quantities above in the pure phases are obtained by setting $\phi_{\alpha}=$ $e_{\alpha}$. For example, we have

$$
\mu_{i}^{\alpha}=\partial_{c_{i}} f_{\alpha}=\partial_{c_{i}} f\left(T, c, e_{\alpha}\right)=L_{i}^{\alpha} \frac{T-T_{i}^{\alpha}}{T_{i}^{\alpha}}+\frac{R}{v_{m}} T\left(\ln \left(c_{i}\right)+1\right)
$$

for the chemical potential of the $i$ th component in the phase $\alpha$.

Now we give some examples for the terms modelling interfacial contributions to the free energy. The simplest form of the gradient energy is

$$
a(\phi, \nabla \phi)=|\nabla \phi|^{2}=\sum_{\alpha=1}^{M}\left|\nabla \phi_{\alpha}\right|^{2} .
$$

However, it has been shown $[17,19,39]$ that gradient energies of the form

$$
a(\phi, \nabla \phi)=\sum_{\substack{\alpha, \beta=1 \\ \alpha<\beta}} A_{\alpha \beta}\left(\phi_{\alpha} \nabla \phi_{\beta}-\phi_{\beta} \nabla \phi_{\alpha}\right),
$$

where $A_{\alpha \beta}$ are convex functions that are homogeneous of degree two, are more convenient with respect to the calibration of parameters in the phase field model to the surface terms in the sharp interface model. A choice that leads to isotropic surface terms is

$$
a(\phi, \nabla \phi)=\sum_{\alpha<\beta} \frac{\tilde{\gamma}_{\alpha \beta}}{\tilde{m}_{\alpha \beta}}\left|\phi_{\alpha} \nabla \phi_{\beta}-\phi_{\beta} \nabla \phi_{\alpha}\right|^{2}
$$

with constants $\tilde{\gamma}_{\alpha \beta}$ and $\tilde{m}_{\alpha \beta}$ that can be related to $\gamma_{\alpha \beta}$ and $m_{\alpha \beta}$ in (21) (cf. [17]). For the bulk potential one may take the standard multiwell potential

$$
w_{s t}(\phi)=9 \sum_{\alpha<\beta} \tilde{m}_{\alpha \beta} \tilde{\gamma}_{\alpha \beta} \phi_{\alpha}^{2} \phi_{\beta}^{2}
$$

or a higher order variant

$$
\tilde{w}_{s t}(\phi)=w_{s t}(\phi)+\sum_{\alpha<\beta<\delta} \gamma_{\alpha \beta \delta} \phi_{\alpha}^{2} \phi_{\beta}^{2} \phi_{\delta}^{2} .
$$

For practical computations the multiobstacle potential yields good calibration properties. It is defined by

$$
w_{o b}(\phi)=\frac{16}{\pi^{2}} \sum_{\alpha<\beta} \tilde{m}_{\alpha \beta} \tilde{\gamma}_{\alpha \beta} \phi_{\alpha} \phi_{\beta}
$$


with a higher order variant

$$
\tilde{w}_{o b}(\phi)=w_{o b}(\phi)+\sum_{\alpha<\beta<\delta} \gamma_{\alpha \beta \delta} \phi_{\alpha} \phi_{\beta} \phi_{\delta}
$$

where $w_{o b}$ and $\tilde{w}_{o b}$ are defined to be infinity whenever $\phi$ is not on the Gibbs simplex $G=\left\{d \in \Sigma^{M}: d_{\alpha} \geq 0\right\}$. We refer to [18] and [19] for a further discussion of the properties of the surface terms.

3.2. Possible choices of the mobility matrix. Here we give an example only for the part of the mobility matrix $\left(L_{i j}\right)_{i, j=0, \ldots, N}$ that defines mass diffusion resulting from chemical potential differences; i.e., we do not specify $L_{i 0}=L_{0 i}$ for $0 \leq i \leq N$. An example for those terms, which in particular define cross effects between mass and energy diffusion, will be given in section 3.4.

If $l_{i}\left(c_{i}, T, \phi\right)$ are the nonnegative bare mobilities of the pure components, we can argue as in [4] to obtain

$$
L_{i j}(T, c, \phi)=l_{i}\left(T, c_{i}, \phi\right)\left(\delta_{i j}-\left(\sum_{q=1}^{N} l_{q}\left(T, c_{q}, \phi\right)\right)^{-1} l_{j}\left(T, c_{j}, \phi\right)\right), \quad 1 \leq i, j \leq N .
$$

To give a simple example, we assume that all bare mobilities are the same constant (e.g., $\left.l_{i}\left(T, c_{i}, \phi\right)=1\right)$. Hence

$$
\left(L_{i j}\right)_{i, j=1}^{N}=i d-\frac{1}{N} \mathbf{1} \otimes \mathbf{1}
$$

where $\mathbf{1}=(1, \ldots, 1)$ and $\otimes$ is the tensor product. Often it is more reasonable to assume that the bare mobilities $l_{i}$ are linear in $c_{i}$, and in the simplest case $\left(l_{i}\left(T, c_{i}, \phi\right)=\right.$ $c_{i}$ ) we obtain

$$
\left(L_{i j}\right)_{i, j=1}^{N}=\left(c_{i}\left(\delta_{i j}-c_{j}\right)\right)_{i, j=1}^{N} .
$$

Choosing a free energy of the form (25) and taking (28) into account, we get the following equations for the concentrations:

$$
\begin{aligned}
\partial_{t} c_{i}=-\nabla \cdot & {\left[L_{i 0} \nabla \frac{1}{T}+\sum_{j=1}^{N} c_{i}\left(\delta_{i j}-c_{j}\right) \nabla\left(-\sum_{\alpha=1}^{M}\left(L_{j}^{\alpha}\left(\frac{1}{T_{j}^{\alpha}}-\frac{1}{T}\right) h\left(\phi_{\alpha}\right)\right)\right.\right.} \\
& \left.\left.-\frac{R}{v_{m}}\left(\ln \left(c_{j}\right)+1\right)\right)\right] \\
=\nabla \cdot & {\left[L_{i 0} \nabla \frac{1}{T}+\sum_{\alpha=1}^{M} \sum_{j=1}^{N} L_{i j} \nabla\left(L_{j}^{\alpha}\left(\frac{1}{T_{j}^{\alpha}}-\frac{1}{T}\right) h\left(\phi_{\alpha}\right)\right)\right]+\frac{R}{v_{m}} \Delta c_{i} . }
\end{aligned}
$$

3.3. Relation to the Penrose-Fife model. In this subsection we will demonstrate that our model includes the model of Penrose and Fife [34] as a special case. In this case there is only one component, and we can neglect the variable $c$. There are two phases, so we will write the equations in terms of the solid fraction $\psi=\phi_{1}$. Then by $(2), \phi_{2}=1-\psi$. 
The first phase, the solid one, is characterized by $\phi=1$; hence $\psi=1$. We assume its free energy density to be

$$
f^{s}=L \frac{T-T_{m}}{T_{m}}-c_{v} T(\ln (T)-1)
$$

where $T_{m}$ is the melting temperature and $L$ the latent heat of the solid-liquid phase transition. The second phase, the liquid one, is characterized by $\phi=e_{2}$; therefore $\psi=0$, and we take the free energy density to be

$$
f^{l}=-c_{v} T(\ln (T)-1) .
$$

We have

$$
f(T, \psi)=L \frac{T-T_{m}}{T_{m}} h(\psi)-c_{v} T(\ln (T)-1)
$$

hence

$$
s(T, \psi)=-\frac{L}{T_{m}} h(\psi)+c_{v} \ln (T)
$$

so that $e(T, \psi)=-L h(\psi)+c_{v} T$. The evolution equation for the energy density yields

$$
c_{v} \partial_{t} T-L h^{\prime}(\psi) \partial_{t} \psi=-\nabla \cdot\left(L_{00} \nabla \frac{1}{T}\right) .
$$

Now we choose $L_{00}=c_{v} K_{2} T^{2}, \lambda(\psi)=L h^{\prime}(\psi) / c_{v}$, and

$$
a(\phi, \nabla \phi)=\frac{c}{2}|\nabla \phi|^{2}=\frac{c}{2}\left(\left|\nabla \phi_{1}\right|^{2}+\left|\nabla \phi_{2}\right|^{2}\right),
$$

where $c=\kappa_{1} c_{v} /(2 \varepsilon)$ for some constant $\kappa_{1}$. Setting $\omega=1, K_{1}=c_{v} /(2 \varepsilon)$ and

$$
s_{0}(\psi)=-\frac{1}{\varepsilon c_{v}} w(\psi, 1-\psi)-\frac{L}{c_{v} T_{m}} h(\psi) .
$$

We arrive at the system

$$
\begin{aligned}
& \partial_{t} \psi=K_{1}\left(\frac{\lambda(\psi)}{T}+s_{0}^{\prime}(\psi)+\kappa_{1} \Delta \psi\right), \\
& \partial_{t} T-\lambda(\psi) \partial_{t} \psi=K_{2} \Delta T
\end{aligned}
$$

which is the model of Penrose and Fife [34, Chapter 6].

3.4. A linearized model. In this subsection we are going to partially linearize our model. This is done in such a way that the evolution equations in the pure phases are linear and they indeed reduce to standard linear diffusion equations. We restrict ourselves to binary systems but a generalization to higher order systems is straightforward.

We denote by $c=c_{1}$ the concentration of the first component; therefore $c_{2}=1-c$. Using that $L$ is symmetric and the algebraic constraints (10), we obtain

$$
L_{01}=L_{10}=-L_{02}=-L_{20} \quad \text { and } \quad L_{11}=L_{22}=-L_{12}=-L_{21} .
$$


Furthermore, we introduce the chemical potential difference

$$
\mu=f_{, c}=f_{, c_{1}}-f_{, c_{2}}=\mu_{1}-\mu_{2} .
$$

Then the conservation laws for energy and concentration read (up to a factor 2 in the last term of the right-hand sides)

$$
\begin{aligned}
& \partial_{t} e=-\nabla \cdot L_{00} \nabla \frac{1}{T}-\nabla \cdot L_{10} \nabla \frac{-f_{, c}}{T}, \\
& \partial_{t} c=-\nabla \cdot L_{10} \nabla \frac{1}{T}-\nabla \cdot L_{11} \nabla \frac{-f_{, c}}{T} .
\end{aligned}
$$

Choosing

$$
L_{11}=D \frac{T}{f_{, c c}}, \quad L_{10}=L_{01}=e_{, c} D \frac{T}{f_{, c c}}, \quad \text { and } \quad L_{00}=e_{, c}^{2} D \frac{T}{f_{, c c}}+K T^{2},
$$

the system (29)-(30) reduces to

$$
\begin{aligned}
& \partial_{t} e=\nabla \cdot\left(K \nabla T+e_{, c} D \nabla c+e_{, c} D \frac{f_{, c \phi}}{f_{, c c}} \nabla \phi\right), \\
& \partial_{t} c=\nabla \cdot\left(D \nabla c+D \frac{f_{, c \phi}}{f_{, c c}} \nabla \phi\right) .
\end{aligned}
$$

Here $K$ and $D$ are coefficients that may depend on $\phi$. Equations (31) and (32) then have to be coupled to the phase field system (12).

We assume as in (27) that the internal energy density is affine linear in the variables $(T, c)$. Then the system $(31)-(32)$ reduces in regions where $\phi$ is constant, i.e., in the pure phases, to (here $K$ and $D$ are constants)

$$
c_{v} \partial_{t} T=\nabla \cdot K \nabla T=K \Delta T, \quad \partial_{t} c=\nabla \cdot D \nabla c=D \Delta c .
$$

Here $c_{v}$ is the specific heat. These are classical linear diffusion equations for temperature (Fourier's law) and concentration (Fick's law).

3.5. Relation to the Caginalp model. If we further linearize the system it can be seen that our model leads to a generalization of the original phase field model [7] to the case of alloy solidification. We consider a three-phase system for a binary alloy. We choose the free energy density

$$
f(T, c, \phi)=\left(\kappa \frac{c}{2}-\sum_{\alpha=1}^{3} L_{1}^{\alpha} \phi_{\alpha}\right) c T-c_{v} T(\ln (T)-1)-\sum_{\alpha=1}^{3} L_{2}^{\alpha} \phi_{\alpha},
$$

where $L_{2}^{\alpha}$ are latent heat coefficients and $L_{1}^{\alpha}$ and $\kappa$, respectively, are coefficients entering the chemical potentials. Then we get

$$
\begin{gathered}
s=-f_{, T}=-\left(\kappa \frac{c}{2}-\sum_{\alpha=1}^{3} L_{1}^{\alpha} \phi_{\alpha}\right) c+c_{v} \ln (T), \\
e=f+T s=c_{v} T-\sum_{\alpha} L_{2}^{\alpha} \phi_{\alpha}, \\
\frac{\mu}{T}=\frac{f_{, c}}{T}=\kappa c-\sum_{\alpha} L_{1}^{\alpha} \phi_{\alpha}, \\
\frac{r_{\alpha}}{T}=\frac{f_{, \phi_{\alpha}}}{T}=-L_{1}^{\alpha} c-\frac{L_{2}^{\alpha}}{T} .
\end{gathered}
$$


Choosing the mobility matrix as in the previous subsection we obtain

$$
\begin{aligned}
& \partial_{t} e=\partial_{t}\left(c_{v} T-\sum_{\alpha} L_{2}^{\alpha} \phi_{\alpha}\right)=\nabla \cdot(K \nabla T), \\
& \partial_{t} c=\nabla \cdot D \nabla\left(\kappa c-\sum_{\alpha} L_{1}^{\alpha} \phi_{\alpha}\right) .
\end{aligned}
$$

For the gradient energy we take the isotropic function $a(\phi, \nabla \phi)=\frac{1}{2} \sum_{\alpha}\left|\nabla \phi_{\alpha}\right|^{2}$. Then the equations for the phase field variables are

$$
\omega \varepsilon \partial_{t} \phi_{\alpha}=\varepsilon \Delta \phi_{\alpha}-\frac{1}{\varepsilon} w_{\phi_{\alpha}}(\phi)+L_{1}^{\alpha} c+\frac{L_{2}^{\alpha}}{T}-\lambda,
$$

where $\lambda$ is the Lagrange multiplier (13). Now we linearize the term $\frac{1}{T}$ in the above equation around a temperature $T_{m}$ to obtain

$$
\omega \varepsilon \partial_{t} \phi_{\alpha}=\varepsilon \Delta \phi_{\alpha}-\frac{1}{\varepsilon} w_{, \phi_{\alpha}}(\phi)+L_{1}^{\alpha} c+L_{2}^{\alpha}\left(\frac{1}{T_{m}}-\frac{1}{T_{m}^{2}}\left(T-T_{m}\right)\right)-\lambda .
$$

The equations for $(T, c)$ are linear and all terms in the equation for $\phi$ are linear except for the term $w_{, \phi_{\alpha}}$. A complete linearization cannot be expected because systems with moving interfaces can never be linear, as can be easily seen for the sharp interface model.

Finally, we note that this simplification of the model leads to a linearized phase diagram; in particular, the magnitude of the jump of the concentration in the sharp interface model is constant for each of the phase boundaries.

3.6. Fields of application. In this paragraph, we comment on the generality of the presented phase field model, on the new features, and on the various different applications to solidification processes, microstructure formation, and polycrystalline grain growth. With the phase field model set up for an arbitrary number of alloy components and phases in a nonisothermal system, the set of governing equations is able to describe the coupled heat and mass diffusion processes as well as the phase transformations in multicomponent systems. Due to the flexibility to choose parameters in the gradient and in the potential free energy, the model consists of enough degrees of freedom to prescribe the physics of each phase boundary and interface separately by defining values for appropriate surface energies $\tilde{\gamma}_{\alpha \beta}$ and for the mobilities $\tilde{m}_{\alpha \beta}$. The model allows for both kinetic and surface energy anisotropies. Different types of anisotropy such as smooth and crystalline expressions corresponding to Wulff shapes with a different number of vertices can be realized in three dimensions. Considering the application point of view, the effect of the type and strength of anisotropy on the growth structure can be investigated. Examples of experimentally observed anisotropic characteristics in eutectic systems are tilted or spiral phase formations and the growth of neighboring eutectic grains.

The phase field variables $\phi_{\alpha}$ can represent different phases and different grains of orientational variants at the same time. Therefore, phenomena such as eutectic grain formation involving different length scales (grains on the larger scale and a eutectic structure on a smaller scale) and interpretations of the nonconserved order parameters can be described using the new model. A main focus of application in future development is the two- and three-dimensional numerical simulation of solidification in multicomponent alloy systems with arbitrary phase diagrams. By choosing the 
specific thermodynamical quantities - the latent heats of fusion $L_{i}^{\alpha}$ and the melting temperatures $T_{i}^{\alpha}$-and by inserting these data as input parameters for the numerical simulations, different types of phase transformations, such as peritectics, eutectics, and monotectics, are modelled. In particular, the stability of ternary eutectic lamellae with phase arrays of different period length and phase permutations will be investigated by phase field simulations in a forthcoming paper. The results of computed structures are compared with a generalization of the classical Jackson-Hunt theory for ternary eutectics. The occurrence of a ternary phase impurity leads to the formation of eutectic colonies. The resulting complex structure is of multiscale type and can also be modelled with the new approach.

4. Relating the models by asymptotic expansions. By matched asymptotic expansions we want to establish the relation between the phase field model and the sharp interface model that were described in section 2. We are going to generalize methods developed by Caginalp and Fife [8], Bronsard, Garcke, and Stoth [6], Garcke and Novick-Cohen [20], and Garcke, Nestler, and Stoth [17]. We restrict ourselves to two space dimensions, i.e., $d=2$, but generalizations are possible.

Since the quantities $(T, \bar{\mu})$ are continuous across a phase boundary it will be convenient to use them in the asymptotic expansions. More precisely we will use the variables $\phi$ and $u=\left(\frac{-1}{T}, \frac{\bar{\mu}_{1}}{T}, \ldots, \frac{\bar{\mu}_{N}}{T}\right)$. Since $f(T, \cdot, \phi)$ is strictly convex and $f(\cdot, c, \phi)$ is strictly concave, we obtain that the mappings

$$
(T, c, \phi) \mapsto(u, \phi) \quad \text { and } \quad(e, c, \phi) \mapsto(u, \phi)
$$

are both invertible and an exchange of variables between these quantities is possible.

We will use the variables $(u, \phi)$ in the asymptotics but the equations can always be reinterpreted with respect to the variables $(T, c, \phi)$ or $(e, c, \phi)$. We write the conservation laws as

$$
\partial_{t} c_{i}(u, \phi)=\nabla \cdot \sum_{j=0}^{N} L_{i j}(u, \phi) \nabla u_{j}, \quad 0 \leq i \leq N,
$$

where we have set $c_{0}=e$.

The phase field equations are

$\omega \varepsilon \partial_{t} \phi=P^{M}\left[\varepsilon\left(\nabla \cdot a_{, X}(\phi, \nabla \phi)-a_{, \phi}(\phi, \nabla \phi)\right)-\frac{1}{\varepsilon} w_{, \phi}(\phi)+u_{0} f_{, \phi}(T(u, \phi), c(u, \phi), \phi)\right]$.

We assume that the matrix $L=\left(L_{i j}\right)_{i, j=0}^{N}$ is strictly positive definite for all arguments on the space

$$
H^{N}:=\left\{d=\left(d_{i}\right)_{i=0}^{N} \in \mathbf{R}^{N+1}: \sum_{i=1}^{N} d_{i}=0\right\}=\mathbf{R} \times T \Sigma^{N} .
$$

In addition, we will frequently make use of the fact that $a$ is homogeneous of degree two in the variable $X$. In particular, we have (cf. [17])

$$
\begin{aligned}
a_{, X}(\phi, \eta X): X & =2 \eta a(\phi, X), \\
a_{, \phi}(\phi, \eta X): X & =\eta^{2} a_{, \phi}(\phi, X), \\
a(\phi, 0) & =0, \\
a_{, X}(\phi, 0) & =0 .
\end{aligned}
$$


4.1. Outer expansion. We expect, based on experiences from numerical simulations, that several phases arise which are separated by diffuse interfaces whose thickness is of order $\varepsilon$. We will see that these phases correspond to the $M$ minima of the potential $w$. In such a phase, away from an interface to another phase, we consider an outer expansion in the bulk region. For a function $b$ in $(t, x)$ we present the ansatz

$$
b_{\text {out }}(t, x)=\sum_{K=0}^{\infty} \varepsilon^{K} b_{\text {out }}^{K}(t, x) .
$$

In this way we expand the variables $u_{j}$ and $\phi_{\alpha}, 0 \leq j \leq N, 1 \leq \alpha \leq M$. For the constraints $\phi \in \Sigma^{M}$ and $u \in H^{N}$ to be satisfied we assume

$$
\begin{array}{ll}
\phi_{\text {out }}^{0} \in \Sigma^{M}, & \phi_{\text {out }}^{K} \in T \Sigma^{M}, \quad K \geq 1, \\
u_{\text {out }}^{K} \in H^{N}, & K \geq 0 .
\end{array}
$$

First we consider the equation for the phase field variables. We expand $P^{M} w_{, \phi}(\phi)$ as

$$
P^{M} w_{, \phi}(\phi)=P^{M} w_{, \phi}\left(\phi_{\text {out }}^{0}\right)+\varepsilon\left(P^{M} w_{, \phi}\right)_{, \phi}\left(\phi_{\text {out }}^{0}\right) \cdot \phi_{\text {out }}^{1}+O\left(\varepsilon^{2}\right) .
$$

To leading order $O\left(\varepsilon^{-1}\right)$ the equation (12) becomes

$$
0=P^{M} w_{, \phi}\left(\phi_{\text {out }}^{0}\right)=w_{, \phi}\left(\phi_{\text {out }}^{0}\right)-\frac{1}{M}\left(\sum_{\alpha=1}^{M} w_{, \phi_{\alpha}}\left(\phi_{\text {out }}^{0}\right)\right) \mathbf{1} .
$$

As we are searching for stable solutions for this equation, $\phi_{\text {out }}^{0}$ is one of the base vectors $\left\{e_{\beta}\right\}_{1 \leq \beta \leq M}$. We can conclude that to leading order the whole domain $\Omega$ is partitioned into phases which are characterized by the $M$ possible values of $\phi_{\text {out }}^{0}$.

The $O(1)$-equations for the conserved variables are $(0 \leq i \leq N)$

$$
\partial_{t} c_{i}\left(u_{\text {out }}^{0}, \phi_{\text {out }}^{0}\right)=\nabla \cdot \sum_{j=0}^{N} L_{i j}\left(u_{\text {out }}^{0}, \phi_{\text {out }}^{0}\right) \nabla u_{j, \text { out }}^{0} .
$$

Boundary conditions for these equations will be obtained by matching with the inner expansion. One should note that we have expanded the coefficients $L_{i j}$ in $\left(u_{\text {out }}^{0}, \phi_{\text {out }}^{0}\right)$ in the same way as $P^{M} w_{, \phi}$ in $\phi_{\text {out }}^{0}$. In phase $\alpha$, i.e., at points where $\phi_{\text {out }}^{0}=e_{\alpha}$, we write $L_{i j}^{\alpha}(u)=L_{i j}\left(u, e_{\alpha}\right)$. Then the $O(1)$-equations become

$$
\partial_{t} c_{i}\left(u_{\text {out }}^{0}, e_{\alpha}\right)=\nabla \cdot \sum_{j=0}^{N} L_{i j}^{\alpha}\left(u_{\text {out }}^{0}\right) \nabla u_{j, \text { out }}^{0} .
$$

Since $c_{0}=e, u_{0}=-\frac{1}{T}$, and $u_{j}=\frac{\mu_{j}}{T}$ we obtain (15) and (16). We note that an upper index in (15) and (16) refers to the phase, whereas an upper index in this section refers to the order in the expansion.

4.2. Inner expansion. Now we consider an interfacial region where two phases meet. Without loss of generality we assume that $\phi_{\text {out }}^{0}=e_{1}$ in one of the outer regions, denoted by $\Omega_{1}$, and $\phi_{\text {out }}^{0}=e_{2}$ in the other one, denoted by $\Omega_{2}$. We assume that these two regions are separated by a family $\left\{\Gamma_{t}\right\}_{t}$ of evolving smooth curves. Let $\psi$ be a smooth function such that $s \mapsto \psi(t, s)$ is an arc-length parametrization of $\Gamma_{t}$. The 
unit tangential vector $\tau(t, x)$ on $\Gamma_{t}$ in $x=\psi(t, s)$ is given by $\tau(t, x)=\partial_{s} \psi(t, s)$, and the unit normal $\nu(t, x)$ on $\Gamma_{t}$ in $x=\psi(t, s)$ is such that $(\nu, \tau)$ is positively oriented. We choose the orientation in the parametrization $\psi$ such that $\nu$ points into $\Omega_{1}$.

Since the parametrization is smooth, it is possible to introduce new space coordinates $(z(t, x), s(t, x))$ in a strip $S$ around $\Gamma_{t}$ in the following way. We define $r(t, x)=d\left(x, \Gamma_{t}\right)$ to be the signed distance between a point $x$ and $\Gamma_{t}$; i.e., $r$ is positive in $\Omega_{1}$ and negative in $\Omega_{2}$. Then the variable $z$ is defined by $z(t, x)=\frac{1}{\varepsilon} r(t, x)$. Let $P_{t}$ be the projection of $S$ onto $\Gamma_{t}$. Then by the smoothness of $\Gamma_{t}$ one can use the strip $S$ narrow enough such that there is exactly one $s(t, x)$ for every $x \in S$ such that $P_{t}(x)=\psi_{t}(s)$. The following holds:

$$
\begin{aligned}
& \nabla_{x} z(t, x)=\frac{1}{\varepsilon} \nu\left(t, P_{t}(x)\right), \\
& \nabla_{x} s(t, x)=\tau\left(t, P_{t}(x)\right)+O(\varepsilon) .
\end{aligned}
$$

In the new variables $(t, z, s)$ we present for some real function $b$ in $(t, x)$ the ansatz

$$
b_{i n}(t, x)=\sum_{K=0}^{\infty} \varepsilon^{K} b_{i n}^{K}(t, z(t, x), s(t, x)) .
$$

Introducing the notation $\nu\left(P_{t}(x)\right)=\nu(t, s(t, x))$ and, similarly, $\tau\left(P_{t}(x)\right)=\tau(t, s(t, x))$, we obtain

$$
\nabla_{x} b_{i n}(t, z(t, x), s(t, x))=\frac{1}{\varepsilon}\left[\partial_{z} b_{i n}(t, z, s)\right] \nu(t, s)+\left[\partial_{s} b_{i n}(t, z, s)\right] \tau(t, s)+O(\varepsilon),
$$

and for some vector field $\vec{b}$ we have

$$
\nabla_{x} \cdot \vec{b}(t, z(t, x), s(t, x))=\frac{1}{\varepsilon}\left(\partial_{z} \vec{b}(t, z, s)\right) \cdot \nu(t, s)+\left(\partial_{s} \vec{b}(t, z, s)\right) \cdot \tau(t, s)+O(\varepsilon) .
$$

Moreover, it follows that

$$
\begin{aligned}
& \partial_{t} z(t, x)=\partial_{t} \frac{1}{\varepsilon} d\left(x, \Gamma_{t}\right)=-\frac{1}{\varepsilon} v\left(P_{t}(x)\right), \\
& \partial_{t} s(t, x)=-v_{\tau}\left(P_{t}(x)\right)+O(\varepsilon),
\end{aligned}
$$

where $v$ is the normal velocity and $v_{\tau}$ the tangential velocity. We note that $v_{\tau}$ depends on the parametrization, whereas $v$ is an intrinsic quantity. This leads to

$$
\frac{d}{d t} b_{i n}^{K}(t, z(t, x), s(t, x))=\partial_{t} b_{i n}^{K}(t, z, s)-\frac{1}{\varepsilon} v \partial_{z} b_{i n}^{K}(t, z, s)-v_{\tau} \partial_{s} b_{i n}^{K}(t, z, s)+O(\varepsilon) .
$$

Now we expand $\phi$ and $u$ in the variables $(t, z, s)$ and we assume

$$
\begin{array}{ll}
\phi_{i n}^{0} \in \Sigma^{M}, & \phi_{i n}^{K} \in T \Sigma^{M}, \quad K \geq 1, \\
u_{i n}^{K} \in H^{N}, \quad K \geq 1, &
\end{array}
$$

to ensure that the constraints on $\phi$ and $u$ are satisfied. Taking a Taylor expansion of $L_{i j}$ around $\left(u_{i n}^{0}, \phi_{i n}^{0}\right)$ and writing $L_{i j}^{0, i n}=L_{i j}\left(u_{i n}^{0}, \phi_{i n}^{0}\right)$, we obtain from the conservation laws for mass and energy to lowest order, i.e., $O\left(\varepsilon^{-2}\right)$,

$$
0=\frac{d}{d z}\left(\sum_{j=0}^{N} L_{i j}^{0, i n} \partial_{z} u_{j, i n}^{0}\right), \quad 0 \leq i \leq N
$$


where we used that $\partial_{z} \nu=0$. Integrating yields

$$
L \partial_{z} u_{i n}^{0}=k
$$

for some vector $k \in \mathbf{R}^{N+1}$. Later, the matching with the outer solution will give $k=0$.

We have

$$
\partial_{z} \nu=0, \quad \partial_{z} \tau=0, \quad \partial_{s} \tau=\kappa \nu, \quad \partial_{s} \nu=-\kappa \tau,
$$

where $\kappa$ is the curvature of $\Gamma_{t}$. Concerning the sign of the curvature we note that for a circle of radius $r$ whose normal is outward oriented (with our orientation the tangent is then running counterclockwise) the curvature is $-1 / r$.

Hence the $O\left(\varepsilon^{-1}\right)$-equations of the conserved quantities are

$$
\begin{aligned}
-v \partial_{z} c_{i}\left(u_{i n}^{0}, \phi_{i n}^{0}\right)= & -\kappa\left(\sum_{j=0}^{N} L_{i j}^{0, i n} \partial_{z} u_{j, i n}^{0}\right)+\frac{d}{d z}\left(\sum_{j=0}^{N} L_{i j}^{0, i n} \partial_{z} u_{j, i n}^{1}\right) \\
& +\frac{d}{d z}\left(\sum_{j=0}^{N}\left(\left(L_{i j}\right)_{, i, i n}^{0, i n} \cdot u_{j, i n}^{1}+\left(L_{i j}\right)_{, \phi}^{0, i n} \cdot \phi_{i n}^{1}\right) \partial_{z} u_{j, i n}^{0}\right)
\end{aligned}
$$

These equations will further simplify when an expression for $u_{i n}^{0}$ has been derived.

Now we consider the equations for the phase field variables. As done in [17] we expand the $a$-terms in $\left(\phi_{i n}^{0}, \partial_{z} \phi_{i n}^{0} \otimes \nu\right)$, the $w$-term in $\phi_{i n}^{0}$, and the $f$-term in $\left(u_{i n}^{0}, \phi_{i n}^{0}\right)$. To leading order $O\left(\varepsilon^{-1}\right)$ we then obtain the equation

$$
0=\frac{d}{d z}\left(P^{M} a_{, X}\left(\phi_{i n}^{0}, \partial_{z} \phi_{i n}^{0} \otimes \nu\right)\right) \nu-P^{M} a_{, \phi}\left(\phi_{i n}^{0}, \partial_{z} \phi_{i n}^{0} \otimes \nu\right)-P^{M} w_{, \phi}\left(\phi_{i n}^{0}\right) .
$$

Multiplying this equation with $\partial_{z} \phi_{i n}^{0} \in T \Sigma^{M}$ gives

$$
0=\frac{d}{d z}\left(a_{, X}\left(\phi_{i n}^{0}, \partial_{z} \phi_{i n}^{0} \otimes \nu\right):\left(\partial_{z} \phi_{i n}^{0} \otimes \nu\right)-a\left(\phi_{i n}^{0}, \partial_{z} \phi_{i n}^{0} \otimes \nu\right)-w\left(\phi_{i n}^{0}\right)\right) .
$$

The equation of order $O(1)$ is

$$
\begin{aligned}
-\omega v \partial_{z} \phi_{i n}^{0}= & \frac{d}{d z}\left[\left(P^{M} a_{, X}\right)_{, \phi} \cdot \phi_{i n}^{1}\right. \\
& \left.+\left(P^{M} a_{, X}\right)_{, X}:\left(\partial_{s} \phi_{i n}^{0} \otimes \tau+\partial_{z} \phi_{i n}^{1} \otimes \nu\right)\right] \nu+\frac{d}{d s}\left(P^{M} a_{, X}\right) \tau \\
& -\left(P^{M} a_{, \phi}\right)_{, \phi} \cdot \phi_{i n}^{1}-\left(P^{M} a_{, \phi}\right)_{, X}:\left(\partial_{s} \phi_{i n}^{0} \otimes \tau+\partial_{z} \phi_{i n}^{1} \otimes \nu\right) \\
& -\left(P^{M} w_{, \phi}\right)_{, \phi} \cdot \phi_{i n}^{1}+P^{M} u_{0, i n}^{0} f_{, \phi}\left(T\left(u_{i n}^{0}, \phi_{i n}^{0}\right), c\left(u_{i n}^{0}, \phi_{i n}^{0}\right), \phi_{i n}^{0}\right),
\end{aligned}
$$

where $w$ and all its derivatives are evaluated in $\phi_{i n}^{0}$ and $a$ and its derivatives in $\left(\phi_{i n}^{0}, \partial_{z} \phi_{i n}^{0} \otimes \nu\right)$.

4.3. Matching and resulting jump conditions. For some quantity $b(t, x)$ we gave by (37) and (40) expansions in bulk regions, respectively, in a strip around an interface between such regions. Now we want to match these expansions in an overlap domain. We will need the matching conditions of order zero and one. For the outer expansions in $\Omega_{1}$ and $\Omega_{2}$ we will use the subscripts $b_{\text {out } 1}$ and $b_{\text {out } 2}$. 
We observe that near $\Gamma_{t}$ we can express the functions $b_{\text {out }}^{K}(t, x)$ in the variables $(t, z, s)$. By expanding in a Taylor series at the point $(0, s(t, x))$ which corresponds to the boundary point $\psi_{t}(s(t, x)) \in \Gamma_{t}\left(\right.$ remember that $z(t, x)=\frac{1}{\varepsilon} r(t, x)$ and $\left.\partial_{r}=\nu \cdot \nabla_{x}\right)$, we obtain

$$
\begin{aligned}
b_{\text {out }}^{K}(t, x) & =b_{\text {out }}^{K}(t, r(t, x), s(t, x)) \\
& =b_{\text {out }}^{K}(t, 0, s(t, x))+r \partial_{r}\left(b_{\text {out }}^{K}\right)(t, 0, s(t, x))+O\left(r^{2}\right) \\
& =b_{\text {out }}^{K}(t, 0, s(t, x))+\varepsilon z\left(\nabla_{x} b_{\text {out }}^{K}(t, 0, s(t, x)) \cdot \nu(t, 0, s(t, x))\right)+O\left(\varepsilon^{2}\right),
\end{aligned}
$$

where $b_{\text {out }}^{K}(t, 0, s)$ and $\nabla_{x} b_{\text {out }}^{K}(t, 0, s)$ mean the evaluation in $\left(t, P_{t}(x)\right)$. We get

$$
b_{\text {out }}(t, x)=b_{\text {out }}^{0}(t, 0, s)+\varepsilon\left(z\left(\nabla_{x} b_{\text {out }}^{0}(t, 0, s) \cdot \nu(t, s)\right)+b_{\text {out }}^{1}(t, 0, s)\right)+O\left(\varepsilon^{2}\right) .
$$

Now we consider an intermediate variable $z_{\varepsilon}=\eta(\varepsilon) z$ for some $z>0$, where $\eta(\varepsilon)$ is some function in $\varepsilon$ in the overlap domain of validity of the two expansions (which we suppose to exist); i.e., $\eta=o(1)$ and $\varepsilon=o(\eta)$. Because of $z=r / \varepsilon$ we have $z_{\varepsilon} \rightarrow \pm \infty$ as $\varepsilon \rightarrow 0$.

We substitute the variable $z$ in our expansions by this intermediate variable $z_{\varepsilon}$ and consider their difference; the expansions of $u$ match if, in the limit as $\varepsilon \rightarrow 0$, the terms of every order $\varepsilon^{K}$ vanish. For the $O(1)$-terms this means

$$
\begin{aligned}
& 0 \stackrel{!}{=} \lim _{\varepsilon \searrow 0}\left(b_{\text {out } 1}^{0}(t, 0, s)-b_{\text {in }}^{0}\left(t, z_{\varepsilon}, s\right)\right)=\lim _{z_{\varepsilon} \rightarrow \infty}\left(b_{\text {out } 1}^{0}(t, 0, s)-b_{\text {in }}^{0}\left(t, z_{\varepsilon}, s\right)\right), \\
& 0 \stackrel{!}{=} \lim _{\varepsilon \nearrow 0}\left(b_{\text {out } 2}^{0}(t, 0, s)-b_{\text {in }}^{0}\left(t, z_{\varepsilon}, s\right)\right)=\lim _{z_{\varepsilon} \rightarrow-\infty}\left(b_{\text {out } 2}^{0}(t, 0, s)-b_{\text {in }}^{0}\left(t, z_{\varepsilon}, s\right)\right),
\end{aligned}
$$

while for the $O\left(\varepsilon^{1}\right)$-terms the matching condition is

$$
\begin{aligned}
& 0 \stackrel{!}{=} \lim _{z_{\varepsilon} \rightarrow \infty}\left(z_{\varepsilon} \nabla_{x} b_{\text {out } 1}^{0}(t, 0, s) \cdot \nu(t, s)+b_{\text {out } 1}^{1}(t, 0, s)-b_{\text {in }}^{1}\left(t, z_{\varepsilon}, s\right)\right), \\
& 0 \stackrel{!}{=} \lim _{z_{\varepsilon} \rightarrow-\infty}\left(z_{\varepsilon} \nabla_{x} b_{\text {out } 2}^{0}(t, 0, s) \cdot \nu(t, s)+b_{\text {out } 2}^{1}(t, 0, s)-b_{\text {in }}^{1}\left(t, z_{\varepsilon}, s\right)\right) .
\end{aligned}
$$

First we apply the matching conditions on the functions $u_{j, i n}^{0}, 0 \leq j \leq N$, solving the differential equations (42). The assumption on $L$ yields

$$
\partial_{z} u_{i n}^{0}=L^{-1} k .
$$

By the matching conditions of order zero, $u_{i n}^{0}$ must be bounded if $|z| \rightarrow \infty$. Then the assumption on $L$ necessarily gives $k=0$ so that $u_{i n}^{0}$ is constant.

Since $u_{\text {in }}^{0}$ is constant, we obtain that $u_{\text {out } 1}^{0}(t, 0, s)=u_{\text {out } 2}^{0}(t, 0, s)$ and hence $u$, and therefore the temperature and the chemical potential differences are in the sharp interface limit continuous across an interface.

Now, due to $\partial_{z} u_{j, i n}^{0}=0$, the $O\left(\varepsilon^{-1}\right)$-equations (44) for the conserved variables simplify to

$$
-v \partial_{z} c_{i}\left(u_{i n}^{0}, \phi_{i n}^{0}\right)=\frac{d}{d z}\left(\sum_{j=0}^{N} L_{i j}\left(u_{i n}^{0}, \phi_{i n}^{0}\right) \partial_{z} u_{j, i n}^{1}\right) .
$$

Integrating with respect to $z$ from $-\infty$ to $\infty$ (or, more correctly, integrating from $-R$ to $R$ and then considering the limit as $R \rightarrow \infty$ ) and using that $v(t, s)$ is independent 
of $z$, we obtain

$$
v\left[c_{i}\left(u_{i n}^{0}, \phi_{i n}^{0}\right)\right]_{z \searrow-\infty}^{z / \infty}=-\left[\sum_{j=0}^{N} L_{i j}\left(u_{i n}^{0}, \phi_{i n}^{0}\right) \partial_{z} u_{j, i n}^{1}\right]_{z \searrow-\infty}^{z / \infty} .
$$

As has been shown in $[8,6]$ the matching conditions of order one for the $b_{j, i n}^{1}$ yield

$$
\partial_{z} b_{j, \text { in }}^{1} \rightarrow \nabla_{x} b_{j, \text { out } 1}^{0} \cdot \nu \quad \text { for } \quad z \rightarrow \infty
$$

and

$$
\partial_{z} b_{j, \text { in }}^{1} \rightarrow \nabla_{x} b_{j, \text { out } 2}^{0} \cdot \nu \quad \text { for } \quad z \rightarrow-\infty,
$$

where the right-hand sides are evaluated in $(t, x)=\left(t, \psi_{t}(s)\right)$ or, in the other coordinates, in $(t, r, s)=(t, 0, s(t, x))$. In fact, these are the boundary values of $\nabla_{x} u_{j, \text { out } \beta}^{0} \cdot \nu$, $\beta \in\{1,2\}$, on $\Gamma_{t}$. After matching for the phase field variables $\phi$ we obtain

$$
\begin{aligned}
v\left[c_{i}\right]_{2}^{1} & =v\left(c_{i}\left(u_{\text {out } 1}^{0}, \phi_{\text {out } 1}^{0}\right)-c_{i}\left(u_{\text {out } 2}^{0}, \phi_{\text {out } 2}^{0}\right)\right)(t, x) \\
& =v\left[c_{i}\left(u_{\text {in }}^{0}, \phi_{\text {in }}^{0}\right)\right]_{z \searrow-\infty}^{z / \infty} \\
& =-\left(\sum_{j=0}^{N} L_{i j}^{0, \text { out } 1} \nabla_{x} u_{j, \text { out } 1}^{0}-L_{i j}^{0, \text { out } 2} \nabla_{x} u_{j, \text { out } 2}^{0}\right)(t, x) \cdot \nu(t, x) \\
& =\left(J_{i}\left(u_{\text {out } 1}^{0}, \phi_{\text {out } 1}^{0}\right)-J_{i}\left(u_{\text {out } 2}^{0}, \phi_{\text {out } 2}^{0}\right)\right)(t, x) \cdot \nu(t, x) \\
& =\left[J_{i}\right]_{2}^{1} \cdot \nu
\end{aligned}
$$

We will refer to this fact as the jump condition for the inner energy density $e=c_{0}$ and the concentrations $c_{i}, 1 \leq i \leq N$.

4.4. Matching and the Gibbs-Thomson relation. In the bulk regions we have $\phi_{\text {out } \beta}^{0}=e_{\beta}, \beta \in\{1,2\}$. Hence for each $s$, we have to solve equation (44) of second order in $z$ with respect to the boundary conditions $e_{1}$ for $z \rightarrow \infty$ and $e_{2}$ for $z \rightarrow-\infty$.

By integrating (45) and using (35), (36) and $w\left(e_{1}\right)=w\left(e_{2}\right)=0$ we obtain

$$
0=a_{, X}\left(\phi_{i n}^{0}, \partial_{z} \phi_{i n}^{0} \otimes \nu\right):\left(\partial_{z} \phi_{i n}^{0} \otimes \nu\right)-a\left(\phi_{i n}^{0}, \partial_{z} \phi_{i n}^{0} \otimes \nu\right)-w\left(\phi_{i n}^{0}\right) .
$$

Using (33) we deduce

$$
a\left(\phi_{i n}^{0}, \partial_{z} \phi_{i n}^{0} \otimes \nu\right)=w\left(\phi_{i n}^{0}\right)
$$

which is known as equipartition of energy. We set

$$
\begin{aligned}
& C_{\alpha \beta}^{0,1}\left([-1,1], \Sigma^{M}\right)= \\
& \left\{p:[-1,1] \rightarrow \Sigma^{M} \mid p \text { Lipschitz continuous, } p(-1)=e_{\alpha} \text { and } p(1)=e_{\beta}\right\},
\end{aligned}
$$

and define the surface entropy for some $e \in \mathbf{R}^{n}$ to be

$$
\gamma_{\alpha \beta}(e)=\inf \left\{2 \int_{-1}^{1} \sqrt{w(p)} \sqrt{a\left(p, p^{\prime} \otimes e\right)}(y) d y \mid p \in C_{\alpha \beta}^{0,1}\right\} .
$$


As shown in [40,17], if a minimizer exists for $e=\nu(t, s)$, then a reparametrization of the minimizer fulfills (44) and, in addition,

$$
\gamma_{2,1}(\nu)=\int_{-\infty}^{\infty}\left(a\left(\phi_{i n}^{0}, \partial_{z} \phi_{i n}^{0} \otimes \nu\right)+w\left(\phi_{i n}^{0}\right)\right) d z .
$$

Now we want to deduce the Gibbs-Thomson law. We multiply the equation (44) for $\phi_{i n}^{0}$ by $\partial_{z} \phi_{i n}^{1} \in T \Sigma^{M}$ and the equation (47) for $\phi_{i n}^{1}$ by $\partial_{z} \phi_{i n}^{0} \in T \Sigma^{M}$. Observe that we can drop the projections $P^{M}$. Then we sum up the two equations and integrate from $-\infty$ to $\infty$ with respect to $z$. Some straightforward calculations together with the matching conditions for the boundary values yield the following solvability condition for equation (47):

$$
\begin{aligned}
-\omega v \int_{-\infty}^{\infty}\left(\partial_{z} \phi_{i n}^{0}(z, s)\right)^{2}= & \frac{d}{d s}\left(\int_{-\infty}^{\infty} a_{, X}\left(\phi_{i n}^{0}(z, s), \partial_{z} \phi_{i n}^{0}(z, s) \otimes \nu(s)\right) \cdot \partial_{z} \phi_{i n}^{0}(z, s) d z\right) \tau(s) \\
& +\int_{-\infty}^{\infty} u_{0, i n}^{0} f_{, \phi}\left(T\left(u_{i n}^{0}, \phi_{i n}^{0}\right) c\left(u_{i n}^{0}, \phi_{i n}^{0}\right), \phi\right) \cdot \partial_{z} \phi_{i n}^{0} d z .
\end{aligned}
$$

Using that $u_{0, \text { in }}^{0}$ and $\bar{u}_{i n}^{0}=\left(u_{1, i n}^{0}, \ldots, u_{N, \text { in }}^{0}\right)$ are independent of $z$, the last term on the RHS of (53) yields

$$
\begin{aligned}
& \int_{-\infty}^{\infty} u_{0, i n}^{0} f_{, \phi}\left(T_{i n}^{0}, c_{i n}^{0}, \phi_{i n}^{0}\right) \cdot \partial_{z} \phi_{i n}^{0} d z \\
= & \int_{-\infty}^{\infty}\left(\frac{d}{d z}\left(u_{0, i n}^{0} f\left(T_{i n}^{0}, c_{i n}^{0}, \phi_{i n}^{0}\right)\right)-u_{0, i n}^{0} f_{, c}\left(T_{i n}^{0}, c_{i n}^{0}, \phi_{i n}^{0}\right) \cdot \partial_{z} c_{i n}^{0}\right) d z \\
= & \int_{-\infty}^{\infty}\left(\frac{d}{d z}\left(u_{0, i n}^{0} f\left(T_{i n}^{0}, c_{i n}^{0}, \phi_{i n}^{0}\right)\right)+\bar{u}_{i n}^{0} \cdot \partial_{z} c_{i n}^{0}\right) d z \\
= & {\left[u_{0, i n}^{0} f\left(T_{i n}^{0}, c_{i n}^{0}, \phi_{i n}^{0}\right)+\bar{u}_{i n}^{0} \cdot c_{i n}^{0}\right]_{z \searrow-\infty}^{z \succ \infty} } \\
= & :\left[u_{0}^{0}\left(f\left(T^{0}, c^{0}, \phi^{0}\right)-f_{, c}\left(T^{0}, c^{0}, \phi^{0}\right) \cdot c^{0}\right)\right]_{2}^{1} .
\end{aligned}
$$

Here we use the abbreviation $T_{i n}^{0}=T\left(u_{i n}^{0}, \phi_{i n}^{0}\right), c_{i n}^{0}=c\left(u_{i n}^{0}, \phi_{i n}^{0}\right), T^{0}=T\left(u^{0}, \phi^{0}\right)$, and $c^{0}=c\left(u^{0}, \phi^{0}\right)$. Finally, as $\left[c^{0}\right] \in T \Sigma^{N}$ we obtain

$$
\int_{-\infty}^{\infty} u_{0, i n}^{0} f_{, \phi}\left(T_{i n}^{0}, c_{i n}^{0}, \phi_{i n}^{0}\right) \cdot \partial_{z} \phi_{i n}^{0} d z=-\left(\frac{\left[f^{0}\right]_{2}^{1}-\mu^{0} \cdot\left[c^{0}\right]_{2}^{1}}{T^{0}}\right)(t, x) .
$$

Calculating the total derivative of $\gamma_{2,1}$, which becomes with (52)

$$
D \gamma_{2,1}(\nu)=\int_{-\infty}^{\infty} a_{, X} \cdot \partial_{z} \phi_{i n}^{0} d z
$$

and setting

$$
m(\nu)=\omega \int_{-\infty}^{\infty}\left(\partial_{z} \phi_{i n}^{0}\right)^{2} d z,
$$

we reduce the solvability condition to (writing $\nabla_{s} \cdot g=\left(\partial_{s} g\right) \cdot \tau$ for the surface divergence of some vector field $g$ on $\Gamma_{t}$ )

$$
m(\nu) v=-\nabla_{s} \cdot D \gamma_{2,1}(\nu)+\frac{\left[f^{0}\right]_{2}^{1}-\mu^{0} \cdot\left[c^{0}\right]_{2}^{1}}{T^{0}}
$$


Considering $\nu$ and $\gamma$ as functions in an angle $\theta \in[0,2 \pi)$, i.e., setting $\nu(\theta)=(\cos (\theta)$, $\sin (\theta)$ ) and $\hat{\gamma}(\theta)=\gamma(\nu(\theta))$, one can derive (see [17])

$$
\nabla_{s} \cdot D \gamma_{2,1}(\nu)=-\left(\hat{\gamma}_{2,1}(\theta)+\hat{\gamma}_{2,1}^{\prime \prime}(\theta)\right) \kappa
$$

with the curvature $\kappa=-\nabla_{s} \cdot \nu$ which may be inserted into the solvability condition to yield

$$
m(\nu) v=\left(\hat{\gamma}_{2,1}(\theta)+\hat{\gamma}_{2,1}^{\prime \prime}(\theta)\right) \kappa+\frac{\left[f^{0}\right]_{2}^{1}-\mu^{0} \cdot\left[c^{0}\right]_{2}^{1}}{T^{0}} .
$$

Finally, the force balance at triple junctions (24) can be derived as in [17]. Therefore, all equations defining the sharp interface model have been derived by asymptotic expansions.

5. Appendix. In this appendix we will show that for the sharp interface model described in section 2 the entropy does not decrease in time. We consider a situation where a bounded domain $\Omega$ is partitioned into $M$ phases $\Omega_{1}(t), \ldots, \Omega_{M}(t)$ which are separated by smooth boundaries $\Gamma_{\alpha \beta}(t)=\bar{\Omega}_{\alpha} \cap \bar{\Omega}_{\beta} \cap \Omega$. For simplicity we restrict ourselves to two space dimensions, but the calculations can also be done in higher dimensions.

Given some domain $R(t) \subset \Omega$ with smooth boundary $\partial R(t)$ and a smooth evolving curve $\Gamma(t) \subset \Omega$ with normal velocity $v$, we will make use of the following transport identities:

$$
\begin{aligned}
& \left.\frac{d}{d t}\left(\int_{\Gamma(t)} \gamma d \mathcal{H}^{1}\right)\right|_{t=t_{0}}=-\int_{\Gamma\left(t_{0}\right)} \gamma \kappa v d \mathcal{H}^{1}+\sum_{\text {endpoints }} \dot{p} \cdot \tau \text { and } \\
& \left.\frac{d}{d t}\left(\int_{R(t)} u d x\right)\right|_{t=t_{0}}=\int_{R\left(t_{0}\right)} \partial_{t} u d x+\int_{\partial R\left(t_{0}\right)} u v d \mathcal{H}^{1}(x)
\end{aligned}
$$

for some smooth function $u=u(t, x)$ and some constant $\gamma ; \kappa$ is the curvature of the interface $\Gamma$, and $\nu$ is the unit normal. By $\dot{p}$ we denote the velocity of the endpoints of $\Gamma$ and by $\tau$ the exterior tangent vector to $\Gamma(t)$ at the endpoints.

Let the evolution in each phase be given by

$$
\partial_{t} e^{q}=-\nabla \cdot J_{0}^{q}, \quad \partial_{t} c_{i}^{q}=-\nabla \cdot J_{i}^{q}, \quad 1 \leq i \leq N, \quad 1 \leq q \leq M,
$$

with the fluxes given in (15) and (16). We assume that the functions are smooth in their domain $\Omega_{q}$ and that the fluxes vanish at the external boundary of $\Omega$. Observe that $-\partial_{t} c=\nabla \cdot J \in T \Sigma^{N}$. Then

$$
\begin{aligned}
\left.\frac{d}{d t}\left(\int_{\Omega(t)} s(e, c) d x\right)\right|_{t=t_{0}} & =\sum_{\alpha} \int_{\Omega_{\alpha}\left(t_{0}\right)} \partial_{t} s(e, c) d x-\sum_{\alpha<\beta} \int_{\Gamma_{\alpha \beta}\left(t_{0}\right)}[s]_{\alpha}^{\beta} v d \mathcal{H}^{1} \\
= & \sum_{\alpha} \int_{\Omega_{\alpha}\left(t_{0}\right)}\left(s, e \partial_{t} e+\sum_{i} s_{, c_{i}} \partial_{t} c_{i}\right) d x-\sum_{\alpha<\beta} \int_{\Gamma_{\alpha \beta}\left(t_{0}\right)}[s]_{\alpha}^{\beta} v d \mathcal{H}^{1} \\
= & -\sum_{\alpha} \int_{\Omega_{\alpha}\left(t_{0}\right)}\left(\frac{1}{T} \nabla \cdot J_{0}+\sum_{i} \frac{-\bar{\mu}_{i}}{T} \nabla \cdot J_{i}\right) d x \\
& -\sum_{\alpha<\beta} \int_{\Gamma_{\alpha \beta}\left(t_{0}\right)}[s]_{\alpha}^{\beta} v d \mathcal{H}^{1}
\end{aligned}
$$




$$
\begin{aligned}
= & \sum_{\alpha} \int_{\Omega_{\alpha}\left(t_{0}\right)} \nabla \frac{1}{T} \cdot J_{0}+\sum_{i} \nabla \frac{-\bar{\mu}_{i}}{T} \cdot J_{i} d x \\
& +\sum_{\alpha<\beta} \int_{\Gamma_{\alpha \beta}\left(t_{0}\right)}\left(\left[\frac{1}{T} J_{0}+\sum_{i} \frac{-\bar{\mu}_{i}}{T} J_{i}\right]_{\alpha}^{\beta} \cdot \nu-[s]_{\alpha}^{\beta} v\right) d \mathcal{H}^{1} .
\end{aligned}
$$

The fact that $L$ is positive semidefinite leads to

$$
\nabla \frac{1}{T} \cdot J_{0}+\sum_{i} \nabla \frac{-\bar{\mu}_{i}}{T} \cdot J_{i} \geq 0
$$

In addition, we make use of the continuity conditions (17), (18) and the jump conditions (19), (20) to obtain

$$
\begin{aligned}
\left.\frac{d}{d t}\left(\int_{\Omega(t)} s(e, c) d x\right)\right|_{t=t_{0}} & \geq \sum_{\alpha<\beta} \int_{\Gamma_{\alpha \beta}\left(t_{0}\right)}\left(\frac{1}{T}[e]_{\alpha}^{\beta} v+\sum_{i} \frac{-\bar{\mu}_{i}}{T}\left[c_{i}\right]_{\alpha}^{\beta} v-\frac{[T s]_{\alpha}^{\beta}}{T} v\right) d \mathcal{H}^{1} \\
& =\sum_{\alpha<\beta} \int_{\Gamma_{\alpha \beta}\left(t_{0}\right)} \frac{[f]_{\alpha}^{\beta}-\sum_{i} \mu_{i}\left[c_{i}\right]_{\alpha}^{\beta}}{T} v d \mathcal{H}^{1} .
\end{aligned}
$$

Furthermore, we have

$$
\left.\frac{d}{d t}\left(-\int_{\Gamma_{\alpha \beta}(t)} \gamma_{\alpha \beta} d \mathcal{H}^{1}\right)\right|_{t=t_{0}}=\int_{\Gamma_{\alpha \beta}\left(t_{0}\right)} \gamma_{\alpha \beta} \kappa v d \mathcal{H}^{1}-\sum_{\text {endpoints }} \dot{p} \cdot \tau_{\alpha \beta} \gamma_{\alpha \beta}
$$

so that we get

$$
\begin{aligned}
\left.\frac{d}{d t} S\right|_{t=t_{0}} & =\left.\frac{d}{d t}\left(\int_{\Omega(t)} s(e, c) d x-\sum_{\alpha<\beta} \int_{\Gamma_{\alpha \beta}(t)} \gamma_{\alpha \beta} d \mathcal{H}^{1}\right)\right|_{t=t_{0}} \\
& \geq \sum_{\alpha<\beta} \int_{\Gamma_{\alpha \beta}\left(t_{0}\right)}\left(\frac{[f]_{\alpha}^{\beta}-\sum_{i} \mu_{i}\left[c_{i}\right]_{\alpha}^{\beta}}{T}+\gamma_{\alpha \beta} \kappa\right) v d \mathcal{H}^{1} \\
& =\sum_{\alpha<\beta} \int_{\Gamma_{\alpha \beta}\left(t_{0}\right)} m(\nu) v^{2} d \mathcal{H}^{1} \geq 0 .
\end{aligned}
$$

In the last equality we used the Gibbs-Thomson relation (21), the fact that the mobility coefficient $m$ is supposed to be positive, the force balance at triple junctions (24), and the fact that in a closed system the interfaces intersect the exterior boundary by a $90^{\circ}$ angle condition (compare [6] and the references therein).

\section{REFERENCES}

[1] R. F. Almgren, Second-order phase field asymptotics for unequal conductivities, SIAM J. Appl. Math., 59 (1999), pp. 2086-2107.

[2] H. W. Alt And I. Pawlow, A mathematical model of dynamics of non-isothermal phase separation, Phys. D, 59 (1992), pp. 389-416.

[3] H. W. Alt and I. Pawlow, On the entropy principle of phase transition models with a conserved order parameter, Adv. Math. Sci. Appl., 6 (1996), pp. 291-376.

[4] J. W. Barrett, J. F. Blowey, and H. Garcke, On fully practical finite element approximations of degenerate Cahn-Hilliard systems, M2AN Math. Model. Numer. Anal., 35 (2002), pp. $713-748$. 
[5] W. J. Boettinger, J. A. Warren, C. Beckermann, and A. Karma, Phase-field simulations of solidification, Ann. Rev. Mater. Res., 32 (2002), p. 163-194.

[6] L. Bronsard, H. Garcke, And B. Stoth, A multi-phase Mullins-Sekerka system: Matched asymptotic expansions and an implicit time discretization for the geometric evolution problem, Proc. Roy. Soc. Edinburgh Sect. A, 128 (1998), pp. 481-506.

[7] G. CaGinalp, Stefan and Hele Shaw type models as asymptotic limits of the phase field equations, Phys. Rev. A, 39 (1989), pp. 5887-5896.

[8] G. Caginalp and P. C. Fife, Dynamics of layered interfaces arising from phase boundaries, SIAM J. Appl. Math., 48 (1988), pp. 506-518.

[9] G. Caginalp And W. XIE, An analysis of phase-field alloys and transition layers, Arch. Ration. Mech. Anal., 142 (1998), pp. 293-329.

[10] J. W. CAHN AND J. E. HilliaRD, Free energy of a nonuniform system I. Interfacial free energy, J. Chem. Phys., 28 (1958), pp. 258-267.

[11] B. Chalmers, Principles of solidification, Krieger, Melbourne, FL, 1977.

[12] L. Q. Chen AND W. YAng, Computer simulation of the domain dynamics of a quenched system with a large number of non-conserved order parameters: The grain-growth kinetics, Phys. Rev. B, 50 (1994), pp. 15752-15756.

[13] L. Q. Chen, Phase-field models for microstructural evolution, Ann. Rev. Mater. Res., 32 (2002), p. 113-140.

[14] D. N. FAN AND L. Q. Chen, Diffuse-interface description of grain boundary motion, Philosophical Magazine Letters, 75 (1997), pp. 187-196.

[15] E. Fried And M. E. Gurtin, Dynamic solid-solid transitions with phase characterized by an order parameter, Phys. D, 72 (1994), pp. 287-308.

[16] H. Garcke and B. Nestler, A mathematical model for grain growth in thin metallic films, Math. Models Methods Appl. Sci., 10 (2000), pp. 895-921.

[17] H. Garcke, B. Nestler, And B. Stoth, On anisotropic order parameter models for multiphase systems and their sharp interface limits, Phys. D, 115 (1998), pp. 87-108.

[18] H. Garcke, B. Nestler, and B. Stoth, A multiphase field concept: Numerical simulations of moving phase boundaries and multiple junctions, SIAM J. Appl. Math., 60 (1999), pp. 295-315.

[19] H. Garcke, B. Nestler, And B. Stoth, Anisotropy in multi-phase systems: A phase field approach, Interfaces Free Bound., 1 (1999), pp. 175-198.

[20] H. Garcke And A. Novick-Cohen, A singular limit for a system of degenerate Cahn-Hilliard equations, Adv. Differential Equations, 5 (2000), pp. 401-434.

[21] W. L. George and J. A. Warren, A parallel $3 D$ dendritic growth simulator using the phasefield method, J. Comput. Phys., 177 (2002), pp. 264-283.

[22] P. HaAsen, Physikalische Metallkunde, 3rd ed., Springer, Berlin, 1994.

[23] A. Karma AND W.-J. RAPPel, Phase-field method for computationally efficient modeling of solidification with arbitrary interface kinetics, Phys. Rev. E, 53 (1996), pp. 3017-3020.

[24] A. Karma AND W.-J. RappeL, Quantitative phase-field modeling of dendritic growth in two and three dimensions, Phys. Rev. E, 57 (1998), pp. 4323-4349.

[25] A. Karma, Phase-field formulation for quantitative modeling of alloy solidification, Phys. Rev. Lett., 87 (2001), p. 115701-1-115701-4.

[26] L. D. Landau and V. I. Ginzburg, K teorii sverkhrovodimosti, Zh. Eksp. Teor. Fiz, 20 (1950), pp. 1064-1082. English translation: On the theory of superconductivity, in Collected Papers of L.D. Landau, D. ter Haar, ed., Pergamon, Oxford, UK, 1965, pp. 626-633.

[27] J. S. LANGer, Models of pattern formation in first-order phase transitions, in Directions in Condensed Matter Physics, World Scientific, Singapore, 1986, pp. 165-186.

[28] J. Lowengrub and L. Truskinovsky, Quasi-incompressible Cahn-Hilliard fluids and topological transitions, Proc. Roy. Soc. London Ser. A, 454 (1998), pp. 2617-2654.

[29] S. Luckhaus, Solidification of Alloys and the Gibbs-Thomson Law, Preprint 335 SFB 256, Universität Bonn, Bonn, Germany, 1994.

[30] G. B. McFadden, A. A. Wheeler, And D. M. Anderson, Thin interface asymptotics for an energy/entropy approach to phase-field models with unequal conductivities, Phys. D, 144 (2000), pp. 154-168.

[31] B. Nestler And A. A. Wheeler, A multi-phase-field model of eutectic and peritectic alloys: Numerical simulation of growth structures, Phys. D, 138 (2000), pp. 114-133.

[32] B. Nestler, A. A. Wheeler, And H. Garcke, Modelling of microstructure formation and interface dynamics, Comp. Mater. Sci., 26 (2003), pp. 111-119.

[33] B. Nestler, A. A. Wheeler, L. Ratke, And C. Stöcker, Phase-field model for solidification of a monotectic alloy with convection, Phys. D, 141 (2000), pp. 133-154. 
[34] O. Penrose And P. C. Fife, Thermodynamically consistent models of phase field type for the kinetics of phase transition, Phys. D, 43 (1990), pp. 44-62.

[35] N. Provatas, N. Goldenfeld, and J. Dantzig, Adaptive mesh refinement computation of solidification microstructures using dynamic data structures, J. Comput. Phys., 148 (1999), pp. 265-290.

[36] M. PlaPP AND A. KARMA, Multiscale random-walk algorithm for simulating interfacial pattern formation, Phys. Rev. Lett., 84 (2000), pp. 1740-1743.

[37] A. Karma, Y. H. Lee, and M. Plapp, Three-dimensional dendrite-tip morphology at low undercooling, Phys. Rev. E, 61 (2000), pp. 3996-4006.

[38] H. M. Soner, Convergence of the phase-field equations to the Mullins-Sekerka problem with kinetic undercooling, Arch. Ration. Mech. Anal., 131 (1995), pp. 139-197.

[39] I. Steinbach, F. Pezolla, B. Nestler, M. Seesselberg, R. Prieler, G. J. Schmitz, and J. L. L. Rezende, A phase field concept for multi phase systems, Phys. D, 94 (1996), pp. $35-147$.

[40] P. Sternberg, Vector-valued local minimizers of nonconvex variational problems, Rocky Mountain J. Math., 21 (1991), pp. 799-807.

[41] B. Sтотн, A sharp interface limit of the phase field equations, one-dimensional and axisymmetric, European J. Appl. Math., 7 (1996), pp. 603-633.

[42] J. D. VAN DER WAALS, The thermodynamic theory of capillarity under the hypothesis of a continuous variation of density, in Konink. Nederl. Akad. Wetensch. Afd. Natuurk. Eerste Reeks, 1 (1893), in Dutch. English translation (with commentary): J. S. Rowlinson, J. Statist. Phys., 20 (1979), pp. 197-244.

[43] A. Visintin, Models of Phase Transition, Birkhäuser, Boston, 1996.

[44] S.-L. Wang, R. F. Sekerka, A. A. Wheeler, B. T. Murray, S. R. Coriell, R. J. Braun, AND G. B. MCFADDEN, Thermodynamically-consistent phase-field models for solidification, Phys. D, 69 (1993), pp. 189-200.

[45] A. A. Wheeler, G. B. McFadden, and W. J. Boettinger, Phase-field model for solidification of a eutectic alloy, Proc. Roy. Soc. London Ser. A, 452 (1996), pp. 495-525. 